\title{
MiniCoRe: A Miniature, Foldable, Collision Resilient Quadcopter
}

\author{
Levent Dilaveroğlu, Onur Özcan \\ Bilkent University, Department of Mechanical Engineering, Ankara, Turkey
}

\begin{abstract}
Collision management strategies are an integral part of micro air vehicle (MAV) operation for flight sustainability. Among them, collision avoidance strategies require enhanced environmental and situational awareness for generating evasive maneuvers and collision-free trajectories. Simpler and more adaptable option is to prepare for collisions and design the physical system with predicted collision patterns in mind. In this work, a mechanically compliant quadcopter design using origami-inspired foldable robotics methods with protective shock absorbing elements has been proposed for a collision resilient quad-rotor MAV. 2D design of the foldable structure and the manufacturing process, including electronic hardware elements and software has been discussed. Our results show that in low speed collisions, the flight of the quadcopter is uninterrupted. The compliant quadcopter can continue flight after impact in near-hover conditions because of the reduction of impact forces due to the increased impact time.

Index Terms - Soft Robot Materials and Design, Soft Robots, Flexible Robots, Miniature Robots, Unmanned Air Vehicles, Micro Air Vehicle.
\end{abstract}

\section{INTRODUCTION}

Compliance in robotics has become high in demand with the increasing understanding of its practical benefits; such as enhanced adaptability to external effects and safer interactions with the environment. Such compliance can easily be achieved with the use of low cost materials and low-barrier manufacturing inspired from origami methods.

Compliance in aerial robotics is a relatively new concept [1], [2], [3], made possible with the emergence of robust aerial systems. Quadcopters with rigid bodies are relatively easier to operate than the compliant ones; the quadcopters with compliant bodies require fast sensors, electronics, and controllers for successful operation. The advantage of compliance is apparent with the interaction of the robot and its surrounding environment. Collisions are constant threats in micro aerial vehicles (MAVs) by the virtue of their mission definitions: to operate in tight spaces and indoor environments. Any disruption in the supply of lift force; such as power outage, malfunction in the rotors, and sensor or controller errors are critical in the absence of redundancy.

As an advantage, MAVs are inherently more resilient to mechanical stresses due to laws of scaling [4]. However, rotors and electronics are more susceptible to damage in the case of an impact, because of the lower quality components available in smaller size. Current technology allows for collision avoidance using integrated proximity sensors or preplanned trajectories to eliminate the risk. Trade-off of these systems is the increase in weight and software complexity. For most applications, where thrust and computational power is in abundance, collision avoidance algorithms provide great

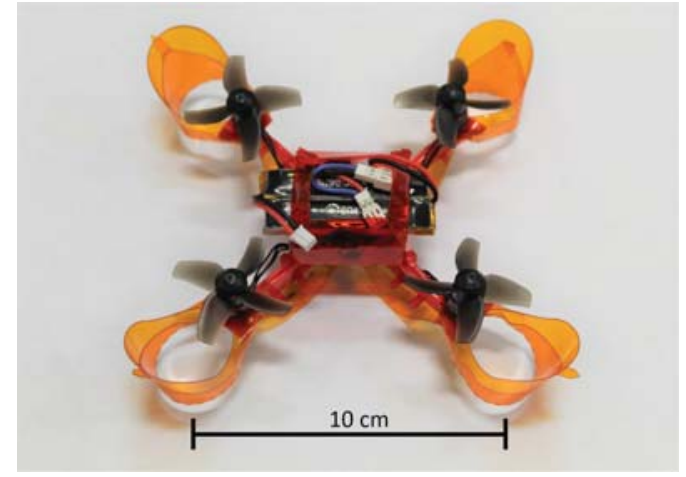

Fig. 1. Miniature, foldable, collision resilient quadcopter, MiniCoRe.

benefit of stable robust operation [5], [6]. However, such methods are often not available in MAVs due to limited sensing capabilities, computational power, battery capacity and thrust output.

Foldable robotics [7], [8], [9], [10], [11] offer a number of advantages for the design and manufacturing of MAVs. Suitability of abundant, lightweight materials allows for lighter structural designs compared to conventional manufacturing methods by trading design complexity. It also offers fast, low cost, batch-prototyping and fabrication. Most important aspect, however, is that foldable robotics offer customized mechanical properties of structures that can be tuned for the purpose [12], [13], [14]. Quadrotor frame experiences rigorous forces compared to its size due to the operation of high speed rotor assembly, which generates lift and control. Body design must provide the quadrotor with reasonable stiffness to hold the rotors firmly, while being able to provide shock reduction with spring-like elasticity.

Collision resilience in MAVs have a presence in the literature providing different perspectives on design and operation. In [1], researchers used a plastic structural material with high elasticity to absorb and redirect the collision energy into deformation. Research in [3], shows a different approach to collision management by covering the quadrotor body with a spherical cage. An origami inspired protective bumper piece has been designed by researchers in [2], which uses similar materials and techniques used in this work.

The contribution of this study is the design and operational demonstration of one of the first miniature quadrotors that is entirely fabricated using foldable robotics methods. This miniature, foldable, collision resilient quadcopter called MiniCoRe (see Fig. 1) is envisioned to be a capable and mechanically compliant flying platform, built using cheap, available materials. Our results demonstrate the effectiveness of foldable structures against impact commonly experienced 
by MAVs. Foldable frame successfully shields the vulnerable rotors and electronics from the impact, while providing structural integrity during flight.

\section{DESIGN}

The designed frame of MiniCoRe is for a quadcopter in $\mathrm{X}$ configuration and weighs around $8 \mathrm{~g}$ (43g including the electronics and the batteries). Propulsion has been provided by four micro outrunner brushless DC motor with approximately $1 \mathrm{~N}$ of thrust output. Frame is constructed using a standard A4-sized cellulose acetate sheet available in many office suppliers. Using the methods of foldable robotics inspired by origami techniques, frame is assembled through pre-planned folds. This design method allows for easy prototyping and customization for different motor and battery types to be incorporated into the frame. Strength of the foldable design method has been demonstrated in the earlier works of the Miniature Robotics Lab, MiniAQ-I/II, a quadrupedal ambulatory robot design [15], [16].

\section{A. Foldable Frame Design}

Design incorporates a number of elements crucial for the flight performance of MiniCoRe. The frame needs to have a high stiffness to withstand the lift forces and reaction torques shown in Fig. 2. Rotor arms have been designed as long and slender beams to increase impact time during collisions. An electronics hub is designed at the center of the body. The hub provides an enclosure to protect electronics while holding the batteries and the arms firmly in their intended configuration. Additional protection against collisions has been provided with rotor bumpers constructed from the same material.

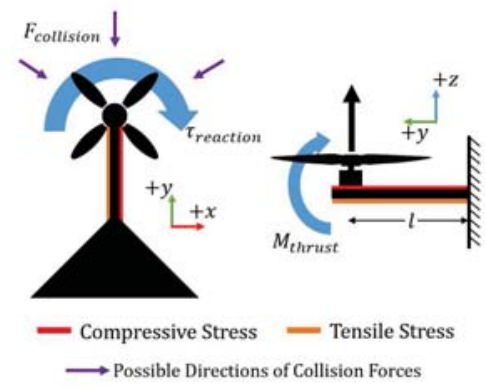

Fig. 2. Forces acting on the rotor arms during flight.

Quadcopter frame has been shaped in the X-Quad configuration with each motor spaced equally. This is advantageous from controls perspective, since the frame becomes identical in pitch and roll axes. Rotors are placed at the tip of arms for maximum moment generation with respect to the center of mass. Longer arms increase the generated moment and moment of inertia, thus arms being longer increases the control authority. Shorter arms require more thrust corrections to steer the quadrotor since the moment arm is reduced, but, shorter arms are lighter and easier to design for required stiffness.

The arms have high bending stiffness in $\mathrm{y}-\mathrm{z}$ plane to restrict the motion of rotors with respect to the body in thrust direction. On the other hand, they have a relatively lower stiffness in $\mathrm{x}-\mathrm{y}$ plane, which causes them to bend significantly upon impact, dissipating the energy of the collision. In order to restrict the motion of arms in y-z plane, a T-fold has been implemented. The T-fold has a side benefit of having a component along the plane of the reaction force, which can restrict this motion with minimal modification of adding a slanted surface to connect both ends of the half $\mathrm{T}$ shape. Two triangular shaped elongated cells are folded from the same piece of cellulose acetate sheet. These cells, when combined, form a larger triangle, increase the overall strength and enable easier folding and manufacturing.

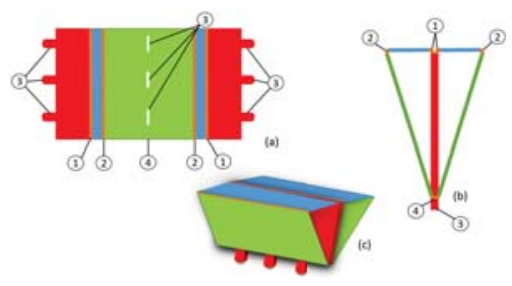

Fig. 3. Folding of the arms. (a) Unfolded cellulose acetate sheet, orange seam lines are perforated with laser for accurate folding. Folding hierarchy is noted with numbers. (b) Cutaway of the folded, load bearing arms; triangular cells can be seen. (c) Overall shape of the folded arms.

Figure 3 shows the folding pattern. Surface shown in red is bearing the load of generated thrust by forming the tail of the T-fold. Blue surfaces restrict red surface from buckling under compression stress. To keep the blue surfaces from collapsing under collision, green surfaces provide another point of support. Locking extensions are inserted into the slots engraved on green surface. These locks block the fold from unwinding and provide the attachment point to the electronics hub. For MiniCoRe, two diagonal arms are required, each supporting two rotors. Arms are joined together to reach X configuration using half-lap joint in the middle point as shown in Fig. 4.

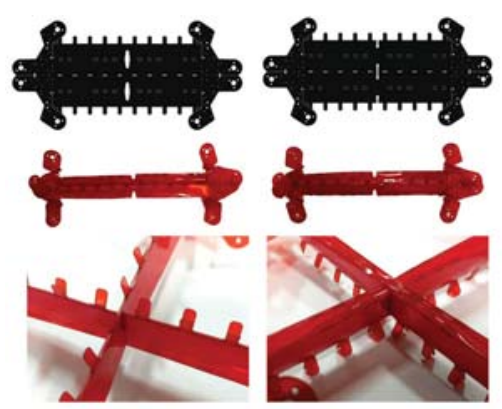

Fig. 4. Two diagonal arms joined together with half-lap joint.

Rotors are required to hold their intended alignment for smooth operation. Misaligned rotors do not cause major disruption thanks to the feedback controller; however, it increases the power consumption due to the loss of thrust. Rotors are held in their place firmly using three $1.2 \mathrm{~mm}$ screws, which connects the rotor base to arm tips. Motor connections can be considered as the most critical part of the design as they sustain the stresses caused by thrust, reaction torques, vibrations, and impact stresses. In order to provide easy access to screw holes and add rigidity to the motor mounts, a three pointed fold is placed at the end of the arms. Three mounting points share the load and direct the stress along the side surfaces of the arm. 
To support the X-Quad arm assembly and to provide housing for the electronics and batteries, the main body housing plays an important role. Main body housing is attached to the assembled arms through the locking extensions of arms. The locking requires pre-stressing of the electronics hub to align the locking extensions with the slots. Locking can be completed by splitting the extensions apart. Pre-stressing of the electronics hub increases the body rigidity by adding another layer of triangular fold over the arms.

Electronics hub is folded around the electronic hardware to provide a steady fixture for flight controller and batteries. Enclosure is an open top rectangular box on the bottom and a half pyramid on the top.

Collision protection ability has been enhanced using bumpers made out of cellulose acetate sheets. Outermost protection is required around the rotors to prevent rotor-blade damage, as the rotor blades are the first points of contact with an obstacle. Protective bumpers absorb the collision energy by elastically deforming, and release the energy by pushing the quadcopter body away from the obstacle, acting as a suspension. The bumper design and potential collision scenarios are shown in Fig. 5.

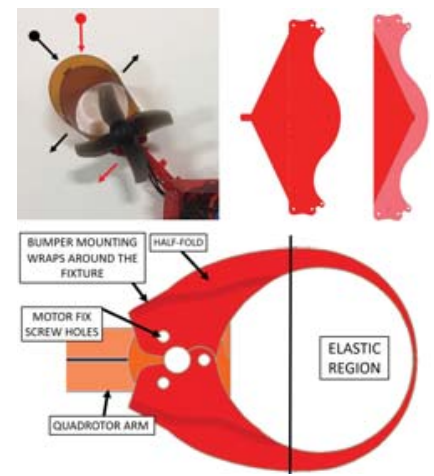

Fig. 5. Collision scenarios and related force reactions(left), Unfolded bumper part (middle), folded and fixed bumper part (right top), bumper and rotor fixture assembly seen from top (right bottom).

Cellulose acetate is formed into a loop mounted around the motor fixture using the motor screws as shown in Fig. 6. An extra layer of cellulose acetate sheet is folded on the bumper part to increase spring coefficient. Mounting point and orientation plays an important role in forming the spring. Bumper mount wraps around the motor fixture from the sides. Half-fold encapsulates the rotor mount with Ushaped folds around the sides. This half-fold rigidly attaches the low stiffness cellulose acetate film to the motor mounts and enables the film to form a directional spring around the rotors. This spring-like section of the bumper absorbs most of the collision energy.

\section{B. Fabrication}

MiniCoRe is easy-to-fabricate thanks to foldable robotics methods used in robot design. The only fabrication required is the engraving of the folding patterns into the cellulose acetate sheet. This is possible using a commercial laser cutting device for fast prototyping and bulk production can

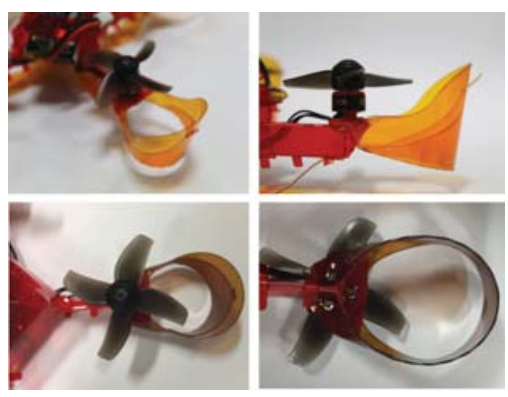

Fig. 6. Implementation of the bumper, mounting point and half-fold is visible.

be achieved by pressing the pattern onto the film using a press machine.

The folding pattern is designed in 2D. The drawn pattern is uploaded to the Universal Laser Systems VLS 3.50 Laser Cutting machine with an A4 cellulose acetate sheet for cutting and engraving operations. Folding lines are marked with a dashed line to decrease material resistance for folding and signal the user where the design should be folded. The pattern includes seven individual unfolded components: the electronics hub, two arms and four bumpers.

\section{Electronics}

To achieve flight, a regulating feed-back control system to provide rotor inputs is essential. On that end, on board computation and sensing capability have to be provided. This need can be satisfied with the commercial flight controller boards. Such boards are capable of fast sensing/computation capability with options to outfit communication suites and extra sensors.

Crazybee F4 is a flight controller that employs 32bit STM32F4 type micro-controller unit integrated with MPU6000 inertial measurement unit, which provides $8 \mathrm{kHz}$ attitude feedback. Along with the flight control unit, $2.4 \mathrm{GHz}$ RF receiver, and a 4-in-1 Electronic Speed Controller unit for $2 \mathrm{~S}$ brushless DC motor control has been packed inside a $4.3 \mathrm{~g}$ package.

The board has been supplied with suitable four Eachine TC0803 brushless DC motors with 15000KV rating. Peak thrust of each motor combined with $40 \mathrm{~mm}$ 4-blade propellers is in average $25 \mathrm{~g}$. Thrust-to-weight ratio of the final quadcopter is approximately 2.3. Two 3.7V 40-80C lithiumpolymer batteries are used to power the MiniCoRe. The electronics used are shown in Fig. 7.

Flight software has been provided by open source Betaflight, which enables users to customize controller settings easily. For the purposes of this work, extensive software modifications are unnecessary, therefore, the flight code has been left unchanged except for stabilization tuning to conduct tests.

\section{Collision Modeling}

In order to formalize the behavior of the foldable frame's deformation under flight stresses and collisions, we should model the system's dynamics. Compliant characteristics of the foldable body and the outer bumpers can be studied 


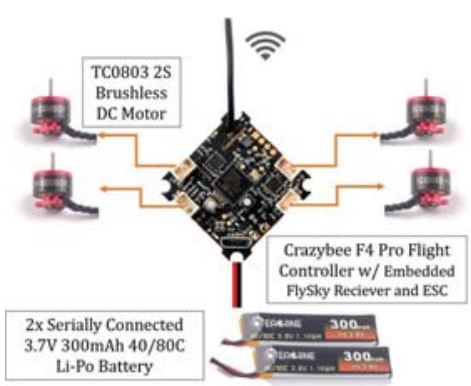

Fig. 7. Setup of the finalized electronic suite equipped with Crazybee F4 Pro.

analytically by modeling the compliance as a spring with energy losses due to friction. By calculating the forces acting upon each component, we can obtain a useful simplified dynamic collision model. For this study, we assume that the collision takes place in the hover position, where pitch and roll angles are zero with the body having an initial velocity vector given in the instance of contact. Additionally, collision surface is perpendicular to MiniCoRe's initial velocity vector. Yaw angle is zero and the direction vector is along the velocity vector. We also assume that the bumper ends are identical and contacting the obstacle at the same instance. Therefore, in the preliminary study, symmetrical conditions are assumed. This simplified model is shown in Fig. 8

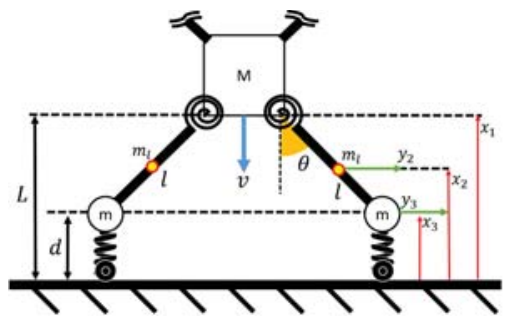

Fig. 8. Structural model of the quadrotor in prescribed collision conditions

The compliance of frame is considered analogous to a spring. It retains the deformation energy with small amount of losses due to the characteristics of the material. Collapsible arms can be modeled as torsional springs complemented with beam equation to obtain the spring constant. Bumpers as well as other structural elements have been assumed to have negligible mass and inertia. It is possible to dissect each mass individually to obtain equations of motion and internal forces.

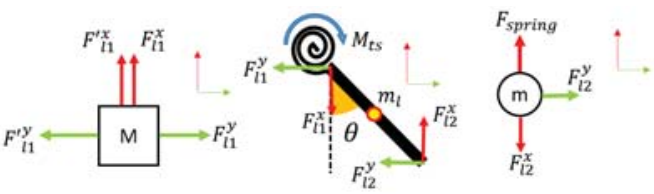

Fig. 9. Free body diagram of the forces acting on the arms.

In this model, $F_{l 1} \mathrm{~s}$ are the link forces. The forces $F^{y}$ shown in Fig. 9 are equal due to symmetry. Therefore, we can write the equation of motion in the $\mathrm{x}$ direction as;

$$
\begin{aligned}
& M \ddot{x_{1}}=F_{l 1}^{x}+F_{l 1}^{\prime x} \\
& M{\ddot{x_{1}}}=2 F_{l 1}^{x} .
\end{aligned}
$$

The equations of motion for the link can be written as;

$$
\begin{gathered}
m_{l} \ddot{x_{2}}=-F_{l 1}^{x}+F_{l 2}^{x} \\
m_{l} \ddot{y_{2}}=-F_{l 1}^{y}-F_{l 2}^{y} \\
I_{l} \ddot{\theta}=\tau_{t s}+F_{l 2}^{y} l \cos \theta-F_{l 2}^{x} l \sin \theta .
\end{gathered}
$$

Figure 9 shows the forces acting on the motor mounts. $F_{b}$ is the spring force generated by the bumpers around the motors. In reality, the bumper has complex spring characteristics, which are directional and non-linear depending on the impact angle. In this model, the springs have been assumed linear. Friction is an important factor in the distribution of the impact energy. In the actual MiniCoRe, thanks to the material properties of cellulose acetate sheets, friction between the wall surface and the bumper is very small and therefore is neglected for this study.

With the aforementioned assumptions, we can write the equation of motion for the motor mount as:

$$
\begin{gathered}
m_{m} \ddot{x_{3}}=F_{b}-F_{l 2}^{x} \\
m_{m} \ddot{y_{3}}=F_{l 2}^{y} \\
F_{b}=k_{b} x \\
\tau_{t s}=k_{t s} \theta,
\end{gathered}
$$

where $k_{t s}$ can be approximated using EulerBernoulli beam equation for the bending of the arms by assuming the interface between the motor and the main body is cantilevered, while the moment is calculated at the fixed point. Using small angle assumptions, we can write:

$$
M=\frac{2 E I}{L^{2}} \theta,
$$

where $E$ is the Young's modulus, $I$ is the second moment of inertia of the beam, and $L$ is the length of the beam. By analogy to rotational spring system, we can deduce:

$$
\begin{aligned}
& \tau=k_{t s} \theta \\
& k_{t s}=\frac{2 E I}{L^{2}} .
\end{aligned}
$$

Using geometric relations, kinematic equations reduce to two degrees of freedom: $x_{3}$ and $\theta$.

$$
\begin{gathered}
x_{1}=x_{3}+l \cos \theta \\
x_{2}=x_{3}+\frac{l}{2} \cos \theta \\
y_{2}=\frac{l}{2} \sin \theta \\
y_{3}=l \sin \theta .
\end{gathered}
$$

Then, we can take the second derivatives of the kinematic relations to acquire:

$$
\begin{aligned}
& \ddot{x_{1}}=\ddot{x_{3}}-l\left(\cos \theta \dot{\theta}^{2}+\sin \theta \ddot{\theta}\right) \\
& \ddot{x_{2}}=\ddot{x_{3}}-\frac{l}{2}\left(\cos \theta \dot{\theta}^{2}+\sin \theta \ddot{\theta}\right) \\
& \ddot{y_{2}}=\frac{l}{2}\left(-\sin \theta \dot{\theta}^{2}+\cos \theta \ddot{\theta}\right) \\
& \ddot{y_{3}}=l\left(-\sin \theta \dot{\theta}^{2}+\cos \theta \ddot{\theta}\right) .
\end{aligned}
$$


Using eqn. 14, we can find the link forces by inserting them into the equations of motion, eqn. 5, 6, and 7 .

$$
\begin{aligned}
\alpha=\cos \theta \dot{\theta}^{2}+\sin \theta \ddot{\theta} \quad \beta=-\sin \theta \dot{\theta}^{2}+\cos \theta \ddot{\theta} \\
F_{l 1}^{x}=\frac{M}{2}\left(\ddot{x_{3}}-\alpha l\right) \\
F_{l 2}^{x}=m_{l}\left(\ddot{x_{3}}-\alpha \frac{l}{2}\right)+F_{l 1}^{x} \\
F_{l 2}^{y}=m_{l} l \beta \\
F_{l 1}^{y}=-F_{l 2}^{y}-m_{l} \beta \frac{l}{2}
\end{aligned}
$$

Using the link force equations, we obtain two ordinary differential equations of $x_{3}$ and $\theta$ as:

$$
\begin{aligned}
& m_{m} \ddot{x_{3}}=k_{b}\left(x_{3}^{0}-x_{3}\right)-m_{l}\left(\ddot{x_{3}}+\beta \frac{l}{2}\right)-\frac{M}{2}\left(\ddot{x_{3}}+\alpha l\right) \\
& I \ddot{\theta}=k_{t} s\left(\theta^{0}-\theta\right)-m_{l} l \sin \theta\left(\ddot{x_{3}}+\frac{l}{2} \beta\right)+m_{m} \alpha l \cos \theta
\end{aligned}
$$

We can impose boundary conditions just before contact as;

$$
\begin{array}{ll}
x_{3}^{0}=0 & \dot{x_{3}^{0}}=v \\
\theta_{0}=\frac{\pi}{4} & \dot{\theta_{0}}=0
\end{array}
$$

We can use a non-linear ODE solver to solve for $x_{3}$ and $\theta$. Inner forces and component accelerations can be calculated to observe the material behavior during impact. The resulting dynamic model can be utilized to obtain the impact force by calculating the deformation time and the peak acceleration.

\section{EXPERIMENTS}

Tests are conducted to demonstrate the performance of MiniCoRe under collision. To evaluate the effectiveness of compliance, experiments are conducted in parallel with a 3D printed rigid quadrotor design using PLA 3D printing material. Velocity and acceleration data are evaluated using an OptiTrack Motion capture system. To compensate for tracking errors during collisions, velocity and acceleration data are retrieved from the experimental footage. First experiment is a free fall test from $1.5 \mathrm{~m}$ shown in Fig. 10. It can be seen that bumpers are guiding the arms to deform in the preplanned configuration, which would decrease the transfer of collision energy on the critical parts and distribute it among multiple points by increasing the contact area momentarily. Stored energy then recalls the arms to their original configuration, while pushing against the ground. Interaction of the arms with the collision surface increases the impact time while reducing the peak acceleration experienced by the electronics hub. Construction material shows its limitations in the construction of the bumpers. As the size of the loop around the propellers increase, bumpers lose stiffness as expected, which render them obsolete.

Figure 11 is an in-flight test with the objective of examining collisions during flight. We expect the quadrotor to continue operation after the collision and depart from the object within manageable attitude limits. Our tests suggest that MiniCoRe can continue flying after impact with a wall

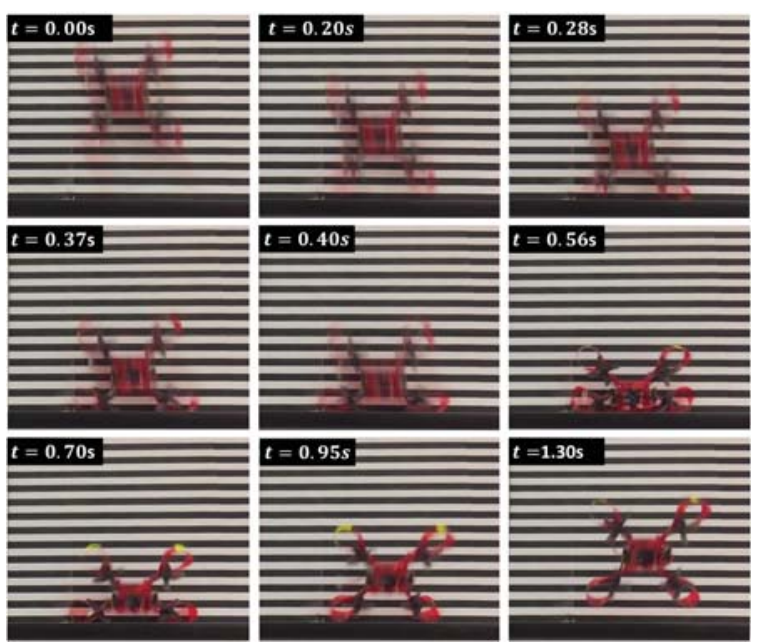

Fig. 10. Drop test from 1.5 meters, showing the bending of rotor arms. The width of the stripes is $1 \mathrm{~cm}$.

if the impact occurred with low speeds (less than $1 \mathrm{~m} / \mathrm{s}$ ) and with a pitch angle that is less than $5^{\circ}$. These parameters are within the limits of normal attitude conditions of near-hover operation like observation and inspection [17]. Bumpers exhibit adequate compression to block propeller collision and to push the frame away from the obstacle.

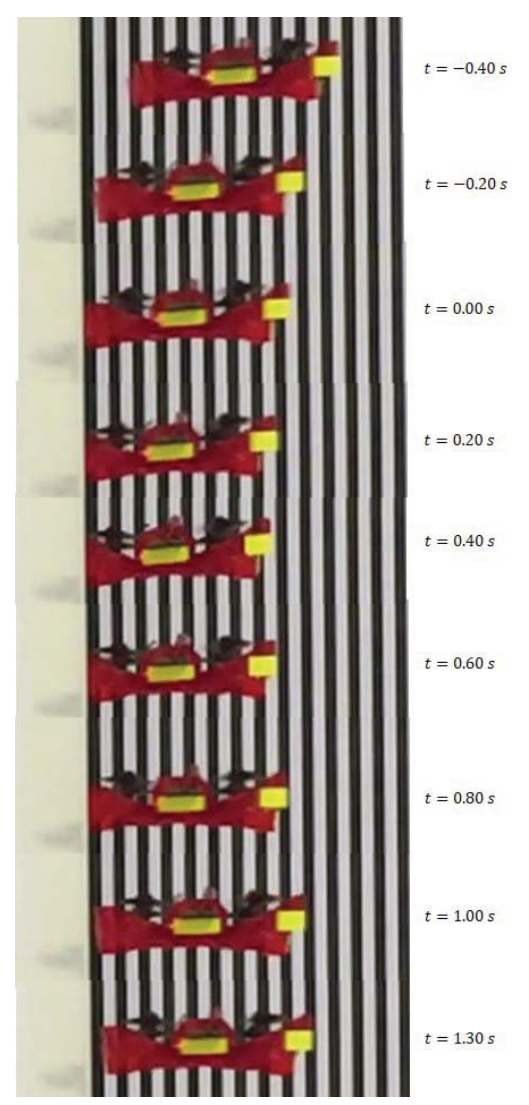

Fig. 11. Powered collision test, width of the stripes is $1 \mathrm{~cm}$

In collision angles of $5^{\circ}-10^{\circ}$, foldable body experiences a flip due to center of mass being above the pivot point (contact point). In order to effectively counter the flip-over, either center of mass should be lowered by placing the battery 


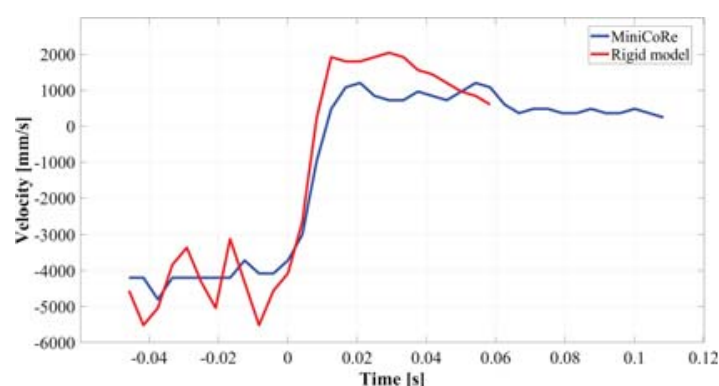

Fig. 12. Unpowered drop test velocities for MiniCoRe and the rigid model

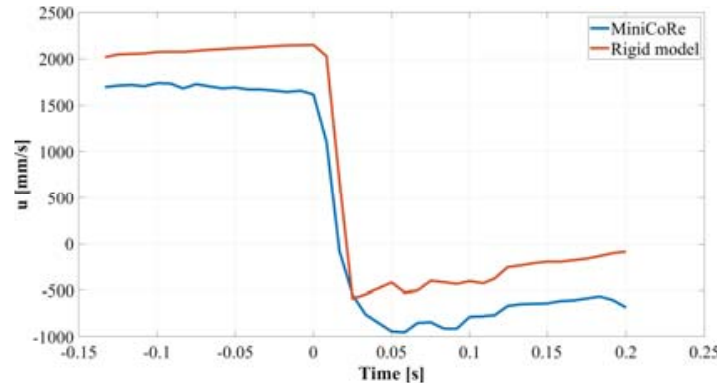

Fig. 13. Powered Impact velocity diagrams for MiniCoRe and the rigid model

mount lower in already packed body hub or bumpers should be larger in order to place the pivot point higher than the center of mass. This has proven to be also problematic due to lowered stiffness of the bumper structure.

As it is illustrated in Fig. 12, for an unpowered free fall test on both MiniCoRe and the rigid model, the impact time for MiniCoRe is about two times the rigid model. This results in an average impact acceleration of about $21 \mathrm{~g}$ for MiniCoRe free drop test and about $38 \mathrm{~g}$ for the rigid model, indicating a greater destructive shock for the rigid one. In addition, in this experiment, the impact restitution factor for MiniCoRe is calculated to be about 0.29, which is lower than the magnitude for the rigid model (calculated as 0.42), meaning $44 \%$ more shock absorption capacity in MiniCoRe.

For the powered impact test, as illustrated in Fig. 13, the impact time for MiniCoRe is almost twice the impact time for the rigid model. Accordingly, the average impact accelerations are about $5 \mathrm{~g}$ and $11 \mathrm{~g}$ for MiniCoRe and the rigid model, respectively. The analysis of data for more than sixty impact experiments for MiniCoRe and the rigid model approves the higher capability of MiniCoRe to survive in impacts.

\section{CONCLUSION AND Future WORKS}

This work presents a miniature quadcopter, named MiniCoRe, which utilizes foldable design features to achieve compliance to withstand impacts. Designed with origami inspired folding techniques, stiffness of the foldable frame is tuned with the collision resilience in mind. The final design of MiniCoRe weighs 43 grams including electronics. Collision resilience characteristics of the compliant frame design has been demonstrated through free fall and powered flight tests which has shown to decrease collision shock and peak acceleration experienced by the body. A simplified model of the frame dynamics is introduced.

As the future work, the MiniCoRe design will be modified to exhibit the same resilient behavior under medium speed and higher attitude angles. Further features will be implemented, such as a landing gear to relieve shock during landings. More detailed collision model will be introduced to compare against the test results. The MiniCoRe will be employed to study multi-body robotic formations for observation and inspection purposes.

\section{ACKNOWLEDGMENTS}

The authors would like to thank Amirali Abazari and Osman Afandiyev for their help in this work. This work is funded by the Scientific and Technological Research Council of Turkey (TUBITAK), Grant number: 118E937.

\section{REFERENCES}

[1] S. Mintchev, S. de Rivaz, and D. Floreano, "Insect-inspired mechanical resilience for multicopters," IEEE Robotics and automation letters, vol. 2, no. 3, pp. 1248-1255, 2017.

[2] P. Sareh, P. Chermprayong, M. Emmanuelli, H. Nadeem, and M. Kovac, "Rotorigami: A rotary origami protective system for robotic rotorcraft," Science Robotics, vol. 3, no. 22, p. eaah5228, 2018.

[3] A. Briod, P. Kornatowski, J.-C. Zufferey, and D. Floreano, "A collision-resilient flying robot," Journal of Field Robotics, vol. 31, no. 4, pp. 496-509, 2014.

[4] R. J. Wood, "The first takeoff of a biologically inspired at-scale robotic insect," IEEE transactions on robotics, vol. 24 , no. 2, pp. 341-347, 2008.

[5] S. Bouabdallah and R. Siegwart, "Full control of a quadrotor," in 2007 IEEE/RSJ International Conference on Intelligent Robots and Systems. Ieee, 2007, pp. 153-158.

[6] N. Gageik, P. Benz, and S. Montenegro, "Obstacle detection and collision avoidance for a uav with complementary low-cost sensors," IEEE Access, vol. 3, pp. 599-609, 2015.

[7] A. M. Hoover and R. S. Fearing, "Fast scale prototyping for folded millirobots," in Robotics and Automation, 2008. ICRA 2008. IEEE International Conference on. Ieee, 2008, pp. 886-892.

[8] E. A. Peraza-Hernandez, D. J. Hartl, R. J. Malak Jr, and D. C. Lagoudas, "Origami-inspired active structures: a synthesis and review," Smart Materials and Structures, vol. 23, no. 9, p. 094001, 2014

[9] A. T. Baisch, O. Ozcan, B. Goldberg, D. Ithier, and R. J. Wood, "High speed locomotion for a quadrupedal microrobot," The International Journal of Robotics Research, vol. 33, no. 8, pp. 1063-1082, 2014.

[10] C. D. Onal, M. T. Tolley, R. J. Wood, and D. Rus, "Origami-inspired printed robots," IEEE/ASME Transactions on Mechatronics, vol. 20, no. 5, pp. 2214-2221, 2015.

[11] C. Karakadıoğlu, M. Askari, and O. Özcan, "Design and operation of miniaq: An untethered foldable miniature quadruped with individually actuated legs," in 2017 IEEE International Conference on Advanced Intelligent Mechatronics (AIM). IEEE, 2017, pp. 247-252.

[12] M. Schenk and S. D. Guest, "Origami folding: A structural engineering approach," in Origami 5: Fifth International Meeting of Origami Science, Mathematics, and Education. CRC Press, Boca Raton, FL, 2011, pp. 291-304

[13] R. J. Lang, Origami design secrets: mathematical methods for an ancient art. AK Peters/CRC Press, 2011.

[14] J. L. Silverberg, A. A. Evans, L. McLeod, R. C. Hayward, T. Hull, C. D. Santangelo, and I. Cohen, "Using origami design principles to fold reprogrammable mechanical metamaterials," Science, vol. 345, no. 6197, pp. 647-650, 2014.

[15] M. Askari, C. Karakadıŏlu, F. Ayhan, and O Özcan, "MinIAQ-II: A miniature foldable quadruped with an improved leg mechanism," in 2017 IEEE International Conference on Robotics and Biomimetics (ROBIO). IEEE, 2017.

[16] M. Askari, "Design, control, modeling, and gait analysis in miniature foldable robotics," Ph.D. dissertation, Bilkent University, 2018.

[17] I. Sa and P. Corke, "Vertical infrastructure inspection using a quadcopter and shared autonomy control," in Field and service robotics. Springer, 2014, pp. 219-232. 